\title{
HAMILTONIAN NORMAL CAYLEY GRAPHS
}

\author{
Juan José Montellano-Ballesteros ${ }^{1}$ \\ AND \\ Anahy Santiago Arguello \\ Instituto de Matemáticas \\ Universidad Nacional Autónoma de México \\ Ciudad Universitaria, México, D.F., C.P. 04510, México \\ e-mail: juancho@im.unam.mx \\ jpscw@hotmail.com
}

\begin{abstract}
A variant of the Lovász Conjecture on hamiltonian paths states that every finite connected Cayley graph contains a hamiltonian cycle. Given a finite group $G$ and a connection set $S$, the Cayley graph $\operatorname{Cay}(G, S)$ will be called normal if for every $g \in G$ we have that $g^{-1} S g=S$. In this paper we present some conditions on the connection set of a normal Cayley graph which imply the existence of a hamiltonian cycle in the graph.
\end{abstract}

Keywords: Cayley graph, hamiltonian cycle, normal connection set.

2010 Mathematics Subject Classification: 05C45, 05C99.

\section{REFERENCES}

[1] N. Alon and Y. Roichman, Random Cayley graphs and expanders, Random Structures Algorithms 5 (1994) 271-284. doi:10.1002/rsa.3240050203

[2] J.A. Bondy and U.S.R. Murty, Graph Theory (Springer, New York, 2008).

[3] J. Bourgain and A. Gamburd, Uniform expansion bounds for Cayley graphs of $S L_{2}\left(\mathcal{F}_{p}\right)$, Ann. of Math. 167 (2008) 625-642. doi:10.4007/annals.2008.167.625

[4] C.C. Chen and N. Quimpo, On strongly hamiltonian abelian group graphs, Combin. Math. VIII (Geelong, 1980) Lecture Notes in Math. 884 (Springer, Berlin-New York, 1981) 23-34.

\footnotetext{
${ }^{1}$ Research supported by PAPIIT- México under project IN107218.
} 
[5] E. Durnberger, Connected Cayley graphs of semi-direct products of cyclic groups of prime order by abelian groups are hamiltonian, Discrete Math. 46 (1983) 55-68. doi:10.1016/0012-365X(83)90270-4

[6] E. Ghaderpour and D. Witte Morris, Cayley graphs on nilpotent groups with cyclic commutator subgroup are hamiltonian, Ars Math. Contemp. 7 (2014) 55-72. doi:10.26493/1855-3974.280.8d3

[7] H.H. Glover, K. Kutnar, A. Malnič and D. Marušič, Hamilton cycles in (2, odd,3)Cayley graphs, Proc. Lond. Math. Soc. 104 (2012) 1171-1197. doi: $10.1112 / \mathrm{plms} / \mathrm{pdr} 042$

[8] H.H. Glover, K. Kutnar and D. Marušič, Hamiltonian cycles in cubic Cayley graphs: the $<2,4 k, 3>$ case, J. Algebraic Combin. 30 (2009) 447-475. doi:10.1007/s10801-009-0172-5

[9] H.H. Glover and D. Marušič, Hamiltonicity of cubic Cayley graph, J. Eur. Math. Soc. 9 (2007) 775-787.

[10] H.H. Glover and T.Y. Yang, A Hamilton cycle in the Cayley graph of the $(2, p, 3)$ presentation of PSL2(p), Discrete Math. 160 (1996) 149-163. doi:10.1016/0012-365X(95)00155-P

[11] K. Keating and D. Witte, On Hamilton cycles in Cayley graphs in groups with cyclic commutator subgroup, Annals of Discrete Math. 27 (1985) 89-102.

[12] K. Kutnar, D. Marušič, D. Morris, J. Morris and P. Šparl, Hamiltonian cycles in Cayley graphs of small order, Ars Math. Contemp. 5 (2012) 27-71. doi:10.26493/1855-3974.177.341

[13] D. Marušič, Hamilonian circuits in Cayley graphs, Discrete Math. 46 (1983) 49-54. doi:10.1016/0012-365X(83)90269-8

[14] I. Pak and R. Radoičić, Hamiltonian paths in Cayley graphs, Discrete Math. 309 (2009) 5501-5508. doi:0.1016/j.disc.2009.02.018

[15] C. Praeger, Finite normal edge-transitive Cayley graphs, Bull. Aust. Math. Soc. 60 (1999) 207-220. doi:10.1017/S0004972700036340

[16] J.J. Rotman, An Introduction to the Theory of Groups, Fourth Edition (SpringerVerlag, New York, 1995).

[17] F. Menegazzo, The number of generator of a finite group, Irish Math. Soc. Bull. 50 (2003) 117-128.

[18] P.E. Schupp, On the structure of hamiltonian cycles in Cayley graphs of finite quotients of the modular group, Theoret. Comput. Sci. 204 (1998) 233-248. doi:10.1016/S0304-3975(98)00041-3

[19] C. Wang, D. Wang and M. Xu, Normal Cayley graphs of finite groups, Sci. China Ser. A 41 (1998) 242-251. doi:10.1007/BF02879042 
[20] D. Witte Morris, Odd-order Cayley graphs with commutator subgroup of order pq are hamiltonian, Ars Math. Contemp. 8 (2015) 1-28.

doi:10.26493/1855-3974.330.0e6

Received 30 December 2017

Revised 12 June 2018

Accepted 19 March 2019 